\title{
Alterations of the base excision repair gene MUTYH in sporadic colorectal cancer
}

\author{
TAKASHI KUNO ${ }^{1}$, NAGAHIDE MATSUBARA ${ }^{1}$, SATOSHI TSUDA $^{1}$, MASAYOSHI KOBAYASHI ${ }^{1}$, \\ MIE HAMANAKA ${ }^{1}$, DAISUKE YAMAGISHI ${ }^{1}$, KIYOSHI TSUKAMOTO ${ }^{1}$, TOMOKI YAMANO ${ }^{1}$, \\ MASAFUMI NODA ${ }^{1}$, HIROKI IKEUCHI ${ }^{1}$, SANGHYUK KIM $^{2}$, KAZUO TAMURA $^{2}$ and NAOHIRO TOMITA ${ }^{1}$ \\ ${ }^{1}$ Division of Lower Gastroenterology, Department of Surgery, Hyogo College of Medicine, Nishinomiya; \\ ${ }^{2}$ Department of Life Science, Faculty of Science and Engineering, Kinki University, Higashiosaka 577-8502, Japan
}

Received February 6, 2012; Accepted April 23, 2012

DOI: $10.3892 /$ or.2012.1836

\begin{abstract}
The base excision repair gene $M U T Y H$ encodes glycosylase which removes adenine residues mispaired with 8-oxo-7,8-dihydro-2'-deoxyguanosine (8-OHG). Biallelic germline mutations of the MUTYH gene are known to cause multiple colorectal adenomas including polyposis and cancer, mostly due to $\mathrm{G}: \mathrm{C} \rightarrow \mathrm{T}: \mathrm{A}$ transversions in proto-oncogenes or tumor suppressor genes. The risk of colorectal cancer (CRC) in monoallelic mutation carriers of $M U T Y H$ is estimated to be higher in comparison with non-carriers. To investigate the possible role in sporadic CRC, we examined alterations of the MUTYH gene including somatic mutations and allelic loss in 101 cases of sporadic CRC, together with the KRAS mutation in some cases. MUTYH mutations in cancer DNA were detected in 3 cases, while mutations were also found in DNA samples from normal tissues, indicating that all were germline mutations. Allelic loss at the MUTYH locus was found in 10 of $51(20.0 \%)$ CRC cases and KRAS mutations were found in 33 of the $101(32.7 \%)$ samples. There was no significant difference in the rate of $\mathrm{G}: \mathrm{C} \rightarrow \mathrm{T}: \mathrm{A}$ transversion in $K R A S$ between cases with allelic loss (1 of 10,10.0\%) and without allelic loss ( 9 of 41, 22.0\%). Investigation of quantitative allelic imbalance at SNP rs3219489 of MUTYH showed that CRC cases with $\mathrm{C}$ allele dominance (minor type corresponding to His) were more frequently detected with $\mathrm{G}: \mathrm{C} \rightarrow \mathrm{T}: \mathrm{A}$ transversions than in
\end{abstract}

Correspondence to: Professor Kazuo Tamura, Department of Life Science, Faculty of Science and Engineering, Kinki University, Kowakae 3-4-1, Higashiosaka 577-8502, Japan

E-mail: tamura@life.kindai.ac.jp

Abbreviations: APC, adenomatous polyposis coli; BER, base excision repair; $\mathrm{CRC}$, colorectal cancer; FAP, familial adenomatous polyposis; LOH, loss of heterozygosity; MAP, MUTYH-associated polyposis; MUTYH; mutY homolog (E. coli); PCR, polymerase chain reaction; RFLP, restriction fragment length polymorphism

Key words: sporadic colorectal cancer, $M U T Y H$ gene, base excision repair gene, loss of heterozygosity those with $\mathrm{G}$ allele dominance (major type corresponding to Gln). In conclusion, somatic alterations of MUTYH in sporadic CRC were rare, similar to other DNA repair genes. However, it is possible that unknown mutations of regions not analyzed in this study and epigenetic changes of the promoter region of MUTYH may contribute to the disease.

\section{Introduction}

Colorectal cancer (CRC) is one of the most prevalent malignancies throughout the world and the third leading cause of cancer death in Japan. Therefore, elucidation of its etiology and development of therapeutic measures are very important (1). To date, a number of studies of CRC development have shown that the etiology is very complex, because of complicated interactions among various environmental factors and a large number of genetic alterations. Genetically, tumorigenesis in CRC is considered to be a multi-step process, including activation of mutations in proto-oncogenes and loss of function of gatekeeper tumor suppressor genes (2). Moreover, DNA repair genes play a critical role in anti-tumorigenesis by maintaining genome integrity (3). The base excision repair pathway is a DNA repair pathway that removes mutations and repairs the constitutional genome via a large amount of coordinated sequential reactions, including detection and processing of damaged nucleotides (4). The MUTYH gene (MIM 604933) is a base excision repair gene that encodes glycosylase involved in removal of adenine residues mispaired with 8-oxo7,8-dihydro-2'-deoxyguanosine (8-OHG), the oxidized form of guanine residue $(5,6)$.

In 2002, a British family with 3 siblings affected by familial adenomatous polyposis without detectable germline mutations in the APC gene was reported to harbor biallelic germline mutations in the MUTYH gene with recessive inheritance of multiple colorectal adenomas and cancer (7-9). This novel entity was termed $M U T Y H$-associated polyposis (MAP). Furthermore, in colorectal tumors derived from MAP patients, somatic $\mathrm{G}: \mathrm{C} \rightarrow \mathrm{T}: \mathrm{A}$ transversions have been frequently detected. These phenomena are compatible with defective adenine excisional repair function of the $M U T Y H$ gene product $(7,10)$. The risk of CRC in monoallelic and biallelic carriers of MUTYH gene mutations was estimated to be increased by 1.68 - and 
Table I. Clinicopathological characteristics of 101 sporadic colorectal cancer cases.

\begin{tabular}{lc}
\hline Characteristics & $\mathrm{n}(\%)$ \\
\hline Gender & \\
Male & $53(52.5)$ \\
Female & $48(47.5)$ \\
Age at surgical treatment (years) & \\
Range & $34-92$ \\
Mean & 67.3 \\
Location & \\
Right-sided colon & $39(38.6)$ \\
Cecum & $9(8.9)$ \\
Ascending colon & $21(20.8)$ \\
Transverse colon & $9(8.9)$ \\
Left-sided colon and rectum & $62(61.4)$ \\
Descending colon & $2(2.0)$ \\
Sigmoid colon & $13(12.9)$ \\
Rectum & $47(46.5)$ \\
Histology & \\
Tubular adenocarcinoma & $88(87.1)$ \\
Well-differentiated & $31(30.7)$ \\
Moderately differentiated & $57(56.4)$ \\
Poorly differentiated adenocarcinoma & $7(6.9)$ \\
Mucinous adenocarcinoma & $6(5.9)$ \\
TNM stage & \\
I & $16(15.8)$ \\
II & $38(37.6)$ \\
III & $34(33.7)$ \\
IV & $13(12.9)$ \\
\hline
\end{tabular}

93-fold, respectively, in a large population-based association study (11).

We hypothesized that somatic alterations of the base excision repair gene $M U T Y H$ might impair the integrity of the tissue genome, leading to sporadic CRC. In this study, we enrolled 101 cases of unselected sporadic CRC, and investigated for mutations in and allelic loss at $M U T Y H$. Furthermore, we investigated $K R A S$ mutations to evaluate the condition of the base excision repair pathway.

\section{Materials and methods}

Patients and tissue samples. The subjects were 101 patients with sporadic CRC who underwent surgery. Their clinicopathological findings are listed in Table I. In this series, we excluded all patients related to familial adenomatous polyposis (FAP) or familial CRC accumulation, such as Lynch syndrome. This study was approved by the Ethical Institutional Review Board of Hyogo College of Medicine and written informed consent was obtained from all subjects prior to enrollment. Following surgical resection, tissue samples were obtained from both cancerous and normal mucosa ( $\sim 30 \mathrm{mg}$ each), then genomic
DNA was extracted using a QIAamp DNA Mini kit (Qiagen, Hilden, Germany).

MUTYH mutation search. We examined DNA sequencing in the promoter region and exons 2, 7, 11, 12 and 13 containing the intron/exon boundary sequences of the MUTYH gene using PCR-DNA direct sequencing. The primers for these analyses were designed based on the flanking intronic sequences presented in published studies (NCBI Reference Sequence: NG_008189.1). Table II shows the primer sequences, corresponding codon numbers, predicted sizes of the PCR fragments, and annealing temperature for PCR. We prepared a standard reaction mixture with HotStarTaq Master Mix kit (Qiagen) and $50 \mathrm{ng}$ of genomic DNA. DNA samples were amplified using a GeneAmp PCR System 9700 (Applied Biosystems, Foster City, CA, USA) with the following cycling parameters: $10 \mathrm{~min}$ at $95^{\circ} \mathrm{C}$, followed by 35 cycles at $94^{\circ} \mathrm{C}$ for $30 \mathrm{sec}$, the appropriate annealing temperature for $30 \mathrm{sec}$, and $72^{\circ} \mathrm{C}$ for $30 \mathrm{sec}$. Extension at $72^{\circ} \mathrm{C}$ during the last cycle was prolonged to $7 \mathrm{~min}$. The PCR products were purified using $1.5 \%$ low-melting temperature agarose gel electrophoresis (12), then subjected to a DNA sequencing reaction with a BigDye Terminator Cycle Sequencing Ready Reaction kit (Applied Biosystems). For analysis, a Genetic Analyzer 3130 DNA sequencing system (Applied Biosystems) was used.

Assessment of loss of heterozygosity $(\mathrm{LOH})$ in MUTYH gene locus. To evaluate allelic loss in MUTYH gene loci, we utilized a loss of heterozygosity ( $\mathrm{LOH}$ ) method with 3 kinds of single nucleotide polymorphisms (SNPs), e.g., rs80323611 in the promoter region, the rs 77542170 of IVS10 $+2 \mathrm{~A} \rightarrow \mathrm{G}$, and the rs3219489 of c. $972 \mathrm{G} \rightarrow \mathrm{C}$ p.Gln324His in exon 12. These SNPs were preliminarily selected on the basis of diversity from 263 kinds of SNPs of the MUTYH gene revealed by investigating 79 disease-free controls in a Japanese population (unpublished data). First, heterozygotes, who were used as informative cases for this LOH study, were selected based on DNA samples from 101 normal mucosal tissues at each SNP using an PCR-RFLP method with appropriate restriction enzymes, such as SacI, $A v a \mathrm{I}$, and BtsI, for each polymorphism site (Table II). Two kinds of alleles were determined by electrophoresis on $2 \%$ agarose gels. Next, DNA samples obtained from cancer tissues of heterozygotes were analyzed using the same method as for normal tissues. The intensity of each allele was converted into a value for area using ImageJ (version 1.46d, NIH), then the ratio of 2 kinds of allele for each case was determined. The ratio between the intensity of the alleles from cancer tissue DNA was compared with that of corresponding normal mucosal tissue. The amount of change in allele intensity was calculated with the following formula:

$$
\frac{\left(\text { Intensity allele } 1_{\text {cancer }}\right) /\left(\text { Intensity allele } 2_{\text {cancer }}\right)}{\left(\text { Intensity allele } 1_{\text {normal tissue }}\right) /\left(\text { Intensity allele } 2_{\text {normal tissue }}\right)}
$$

If a denominator was assigned to one stable allele that showed no intensity changes in both normal and cancer tissue samples, the amount of allelic loss of the other allele in the cancer tissue was estimated. When a score was $<0.6(40 \%$ reduction in intensity of 1 allele from cancer), the case was 
Table II. PCR analysis conditions.

\begin{tabular}{|c|c|c|c|c|c|}
\hline Region & SNP & Primer sequences & $\begin{array}{l}\text { Annealing } \\
\text { temperature }\left({ }^{\circ} \mathrm{C}\right)\end{array}$ & $\begin{array}{l}\text { Predicted } \\
\text { fragment } \\
\text { size (bp) }\end{array}$ & $\begin{array}{l}\text { PCR-RFLP } \\
\text { assay }\end{array}$ \\
\hline \multicolumn{6}{|l|}{ MUTYH } \\
\hline Promoter & rs80323611 & $\begin{array}{l}\text { 5'-AAGGCCTCAAAATTTGGCCT-3' } \\
\text { 5'-TCATGGCCAATGAGCCTTG-3' }\end{array}$ & 60 & 525 & SacI digest \\
\hline Exon 2 & & $\begin{array}{l}\text { 5'-TGGAGGTGAGAGCCACCCTA-3' } \\
\text { 5'-GATTATAAGACACCCAAGACTCCTGG-3' }\end{array}$ & 60 & 221 & - \\
\hline Exon 7 & & $\begin{array}{l}\text { 5'-TGGAGGTGAGAGCCACCCTA-3' } \\
\text { 5'-GATTATAAGACACCCAAGACTCCTGG-3' }\end{array}$ & 60 & 213 & - \\
\hline $\begin{array}{l}\text { IVS10 } \\
\text { and exon } 11\end{array}$ & rs77542170 & $\begin{array}{l}\text { 5'-CTGCCCTATGACACTCAACCC-3' } \\
\text { 5'-TCAGGTTAGAGGAAGAACTGGAATG-3' }\end{array}$ & 60 & 176 & Ava I digest \\
\hline Exon 12 & rs3219489 & $\begin{array}{l}\text { 5'-CCTAAAGCCCTCTTGGCTTGA-3' } \\
\text { 5'-TGTTACTCATGCCACTGCCCT-3' }\end{array}$ & 60 & 279 & BtsI digest \\
\hline Exon 13 & & $\begin{array}{l}\text { 5'-ACCCCTGCCTGGCTGC-3' } \\
\text { 5'-GTTCAAATAGGCCTGTGGATATAGC-3' }\end{array}$ & 60 & 231 & - \\
\hline \multicolumn{6}{|l|}{$K R A S$} \\
\hline Exon 2 & & $\begin{array}{l}\text { 5'-ACTGAATATAAACTTGTGGTAGTTGGCCCT-3' } \\
\text { 5'-AACAAGATTTACCTCTATTGTTGGATCA-3' }\end{array}$ & 55 & 120 & \\
\hline
\end{tabular}

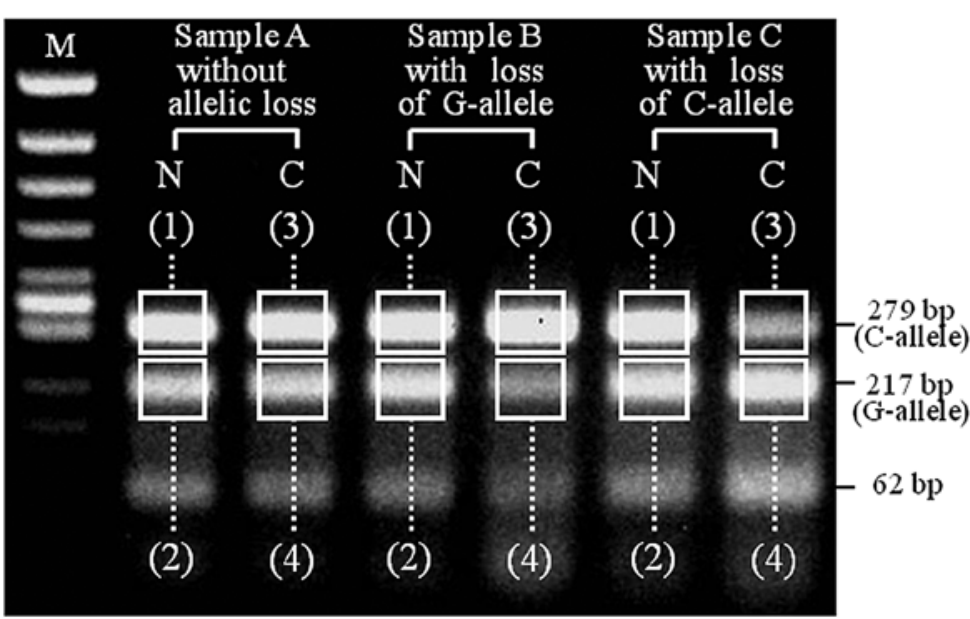

Calculating formula

(i) If a signal of G-allele decreases such as sample B,

Residual allele-intensity

$$
=\frac{(4) /(3)}{(2) /(1)} \times 100(\%)
$$

(ii) If a signal of C-allele decreases such as sample C, Residual allele-intensity

$$
=\frac{(3) /(4)}{(1) /(2)} \times 100(\%)
$$

Figure 1. Representative images from LOH analysis of rs3219489. PCR assays were performed for all CRC samples. PCR products were observed as DNA fragments of $279 \mathrm{bp}$ and digested using BtsI. Reaction solutions were applied to 2\% agarose gel electrophoresis, then 3 kinds of patterns were observed according to the combination of signals among 279, 217 and $62 \mathrm{bp}$. We selected 51 samples with a heterozygous pattern (informative sample). Using the informative samples, genomic DNA derived from both normal mucosal and cancer tissues were analyzed in a pairwise manner. The intensities of the C-allele with $279 \mathrm{bp}$ and the G-allele with $217 \mathrm{bp}$ were scanned and measured as values for the areas using ImageJ (version 1.4.6d). Using a stable allele from both normal and cancer tissues, the intensity of the other side allele from cancer tissue was estimated using a calculation formula (right-sided). Lane M, DNA size marker; $\phi X 174-H a e I I I$ digested; N, derived from normal mucosal tissue; C, derived from cancer tissue; (1), intensity of C-allele from normal tissue; (2), intensity of G-allele from normal tissue; (3), intensity of C-allele from cancer tissue; and (4), intensity of G-allele from cancer tissue

judged as LOH positive (existence of allelic loss) according to Jernvall's model (13). Fig. 1 shows 3 representative patterns; a case without either allelic loss (sample A), a case with a reduction (loss) of the G-allele (sample B), and a case with reduction (loss) of the C-allele (sample C).

KRAS mutation search. To evaluate the state of the base excision repair pathway, whose function might change by alterations of $M U T Y H$, a $K R A S$ mutation search for codon 12 and 13 in exon 2 was performed using a PCR-DNA sequencing method with appropriate primers (Table II).

Statistical analysis. Associations between various biological conditions and clinicopathological factors were compared using a $\chi^{2}$ test, with P-values of $<0.05$ considered to be statistically significant. 


\section{Results}

As a result of sequencing analyses of 6 regions of the MUTYH gene in 101 sporadic CRC cases, we detected 3 kinds of mutations, including 2 missense mutations and 1 nonsense mutation (Table III). The missense mutations, c.53C $\rightarrow$ T p.Pro18Leu and c.74G $\rightarrow$ A p.Gly25Asp, were previously registered in the Human Gene Mutation Database (HGMD) as accession nos.\# CM083727 and CM083730, respectively. On the contrary, no new deleterious mutations were detected, except for SNPs already registered in the dbSNP of the National Center for Biotechnology Information (NCBI). The non-sense mutation, c. $55 \mathrm{C} \rightarrow \mathrm{T}$ p.Arg19X, has not yet been registered in HGMD with regard to germline or mutations. Therefore, this variant is considered to be an unreported novel mutation. These were identified germline mutations and not acquired events, because that same mutations were also found in DNA samples from normal mucosal tissues. Two patients who had dual missense mutations (compound heterozygous) were found to be not affected by colorectal polyposis even after additional detailed history taking. In addition, 2 common mutations of $M U T Y H$ in Caucasians described previously, c.494A $\rightarrow$ G p.Tyr165Cys in exon 7 and c.1145G $\rightarrow$ A p.Gly382Asp in exon $13(7,8)$, were not detected in this study.

By detection of heterozygotes using 3 SNPs, rs80323611 in the promoter region, rs77542170 at the IVS10 accepter site +2 , and rs3219489 at the third letter of codon 324, we found 8,7 , and 51 , respectively, informative samples for an LOH study (Table IVA). Using these informative cases, an LOH study was carried out, however, no significant changes of allelic intensity in the 2 former groups were found in any samples and they were evaluated to be absent from allelic loss (LOH-negative) (Table IVA). On the other hand, 10 (19.6\%) of 51 CRC samples derived from heterozygotes at rs3219489 clearly had allelic loss (Table IVA; Fig. 2). In a comparison between 10 CRC cases with allelic loss and 41 without allelic loss at the site, we found that the state of allelic loss of MUTYH was not correlated with gender ratio, mean age at onset, or stage of cancer progression, such as involvement of lymph nodes or distant organs. However, with regard to location of the CRC occurrence, all cases with allelic loss emerged in the left-sided colon and rectum, in contrast to $18(43.9 \%)$ of 41 without allelic loss CRC, which developed in the right-sided colon (Table IVB). In addition, a $\chi^{2}$ test confirmed that the 2 groups of CRC cases distinguished by biological features had a significant difference regarding the MUTYH gene ( $\mathrm{P}=0.025$, Yates correction). In terms of histopathological findings, all CRC cases with allelic loss had characteristic tubular formation and showed a tendency to be different from allelic stable cases, though it was not significant.

KRAS mutations were detected in 33 of 101 (32.7\%) CRC samples (Table V). These contained 5 kinds of missense mutation, which were distributed as follows: $3(3.0 \%)$ of c. $34 \mathrm{G} \rightarrow \mathrm{T}$ p.Gly12Cys, $10(9.9 \%)$ of c.35G $\rightarrow$ A p.Gly12Asp, $13(12.9 \%)$ of c.35G $\rightarrow \mathrm{T}$ p.Gly12Val, $2(2.0 \%)$ of c. $35 \mathrm{G} \rightarrow \mathrm{C}$ p.Gly12Ala, and 5 of c.38G $\rightarrow$ A p.Gly13Asp. A Gly12Asp mutation, one of the $\mathrm{G}: \mathrm{C} \rightarrow \mathrm{T}: \mathrm{A}$ transversions in $K R A S$, was found in 1 of the $3(33.3 \%)$ CRC cases with biallelic or monoallelic germline mutations in the MUTYH gene. In the 
Table IV. LOH analysis of the MUTYH locus and comparison of CRC cases with or without allelic loss at rs3219489.

A, Characteristics of informative cases in the LOH analysis of the MUTYH locus

\begin{tabular}{lccc}
\hline Polymorphic loci & $\begin{array}{c}\text { rs8023611 } \\
\text { promoter-64G } \rightarrow \mathrm{A}(\%)\end{array}$ & $\begin{array}{c}\text { rs77542170 } \\
\text { IVS10 as-2A } \rightarrow \mathrm{G}(\%)\end{array}$ & $\begin{array}{r}\text { rs3219489 } \\
\mathrm{c} .972 \mathrm{G} \rightarrow \mathrm{C} \text { p.Gln324His }(\%)\end{array}$ \\
\hline $\begin{array}{l}\text { Informative case (heterozygote) } \\
\text { Allelic loss (LOH positive) }\end{array}$ & 8 & 7 & 51 \\
& $0(0.0)$ & $0(0.0)$ & $10(19.6)$ \\
\hline
\end{tabular}

B, Comparison of characteristics of CRC and allelic loss of MUTYH

rs3219489 c. $972 \mathrm{G} \rightarrow \mathrm{C}$ p.Gln324His

Characteristics

(LOH) Positive (\%)

(LOH) Negative (\%)

\begin{tabular}{|c|c|c|}
\hline No. of cases & 10 & 41 \\
\hline Male/Female & $5 / 5$ & $20 / 21$ \\
\hline Mean age (years old) & 66.4 & 67.5 \\
\hline \multicolumn{3}{|l|}{ Location } \\
\hline Right-sided colon & $0(0.0)^{\mathrm{a}}$ & $18(43.9)^{2}$ \\
\hline Cecum & $0(0.0)$ & $6(14.6)$ \\
\hline Ascending colon & $0(0.0)$ & $6(14.6)$ \\
\hline Transverse colon & $0(0.0)$ & $6(14.6)$ \\
\hline Left-sided colon and rectum & $10(100)^{\mathrm{a}}$ & $23(56.1)$ \\
\hline Descending colon & $0(0.0)$ & $1(2.4)$ \\
\hline Sigmoid colon & $1(10.0)$ & $7(17.1)$ \\
\hline Rectum & $9(90.0)$ & $15(36.6)$ \\
\hline \multicolumn{3}{|l|}{ Histology } \\
\hline Tubular adenocarcinoma & $10(100)$ & $36(87.8)$ \\
\hline Well-differentiated & $4(40.0)$ & $16(39.0)$ \\
\hline Moderately differentiated & $6(60.0)$ & $20(48.8)$ \\
\hline Poorly differentiated adenocarcinoma & $0(0.0)$ & $4(9.8)$ \\
\hline Mucinous adenocarcinoma & $0(0.0)$ & $1(2.4)$ \\
\hline \multicolumn{3}{|l|}{ TNM stage } \\
\hline I & $2(20.0)$ & $7(17.1)$ \\
\hline II & $2(20.0)$ & $13(31.7)$ \\
\hline III & $6(60.0)$ & $15(36.6)$ \\
\hline IV & $0(0.0)$ & $6(14.6)$ \\
\hline$K R A S$ mutations & $2(20.0)$ & $17(41.5)$ \\
\hline
\end{tabular}

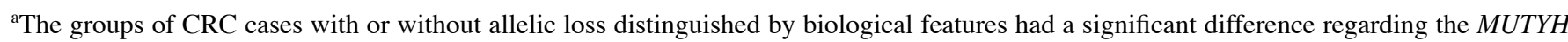
gene $(\mathrm{p}=0.025$, Yates correction).

51 informative cases used in the LOH study, KRAS mutations at rs3219489 were detected in 19 (37.3\%). Furthermore, KRAS mutations were found in $2(20.0 \%)$ of 10 CRC cases with allelic loss (Tables IVB and V). Both c.34G $\rightarrow$ T p.Gly12 and c.35G $\rightarrow$ T p.Gly12Val, shown as underlined letters in Table V, were predicted to be associated with a defect of base excision repair function represented by $\mathrm{G}: \mathrm{C} \rightarrow \mathrm{T}: \mathrm{A}$ transversion of KRAS and found in $16(15.8 \%)$ of all cases. Moreover, $\mathrm{G}: \mathrm{C} \rightarrow \mathrm{T}: \mathrm{A}$ transversions of $K R A S$ were detected in
10 of 51 (19.6\%) CRC cases in which the LOH condition was evaluated with regard to the allelic condition at the $M U T Y H$ locus. Furthermore, $\mathrm{G}: \mathrm{C} \rightarrow \mathrm{T}: \mathrm{A}$ transversion of $K R A S$ was detected in only $1(10.0 \%)$ of 10 cases with allelic loss, whereas it was seen in $9(22.0 \%)$ of 41 CRC cases without allelic loss. In addition, in regard to the quantitative imbalance between both alleles at rs3219489, all mutations and $\mathrm{G}: \mathrm{C} \rightarrow \mathrm{T}: \mathrm{A}$ transversions in $K R A S$ were detected in $11(37.9 \%)$ and $4(13.8 \%)$, respectively, of 29 cases with a G-allele 
Table V. KRAS mutations in CRC.

\begin{tabular}{|c|c|c|c|c|c|c|}
\hline & \multicolumn{4}{|c|}{ Codon12 (GGT) } & \multirow{2}{*}{ 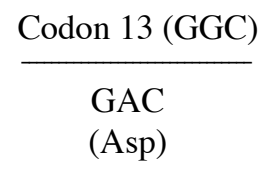 } & \multirow[b]{2}{*}{ Total } \\
\hline & $\frac{\text { TGT }}{\text { (Cys) }}$ & $\begin{array}{l}\text { GAT } \\
\text { (Asp) }\end{array}$ & $\frac{\text { GTT }}{\text { (Val) }}$ & $\begin{array}{l}\text { GCT } \\
\text { (Ala) }\end{array}$ & & \\
\hline All CRC cases $(n=101)$ & $3(3.0)$ & $10(9.9)$ & 13 (12.9) & $2(2.0)$ & $5(5.0)$ & $33(32.7)$ \\
\hline CRC with $M U T Y H$ mutation $(\mathrm{n}=3)$ & $0(0.0)$ & $1(33.3)$ & $0(0.0)$ & $0(0.0)$ & $0(0.0)$ & $1(33.3)$ \\
\hline Informative CRC cases at rs3219489 $(n=51)$ & $1(2.0)$ & $5(9.8)$ & $9(17.6)$ & $1(2.0)$ & $3(5.9)$ & $19(37.3)$ \\
\hline $\mathrm{CRC}$ with allelic loss at $M U T Y H$ locus $(\mathrm{n}=10)$ & $0(0.0)$ & $1(10.0)$ & $1(10.0)$ & $0(0.0)$ & $0(0.0)$ & $2(20.0)$ \\
\hline CRC without allelic loss at $M U T Y H$ locus $(\mathrm{n}=41)$ & $1(2.4)$ & $4(9.8)$ & $8(19.5)$ & $1(2.4)$ & $3(7.3)$ & $17(41.5)$ \\
\hline
\end{tabular}

Underlined letters correspond to $\mathrm{G}: \mathrm{C} \rightarrow \mathrm{T}: \mathrm{A}$ transversions.

Table VI. Correlation of allelic imbalance with G:C $\rightarrow \mathrm{T}$ :A transversions in 51 informative cases.

Allele imbalance at rs3219489

G-allele dominant condition

(relative reduction of $\mathrm{C}$-allele)

$(n=29)(\%)$
C-allele dominant condition (relative reduction of $\mathrm{G}$-allele) $(n=22)(\%)$
CRC with KRAS mutation

$\mathrm{CRC}$ with $\mathrm{G}: \mathrm{C} \rightarrow \mathrm{T}:$ A transversion in $K R A S$

Ratio of CRC cases with $\mathrm{G}: \mathrm{C} \rightarrow \mathrm{T}:$ A transversions

and those with $K R A S$ mutations

$\begin{array}{cc}11(37.9) & 8(36.4) \\ 4(13.8) & 6(27.3) \\ 36.4 & 75.0\end{array}$

G-allele, major variant and corresponding to Gln; C-allele, minor variant corresponding to His.

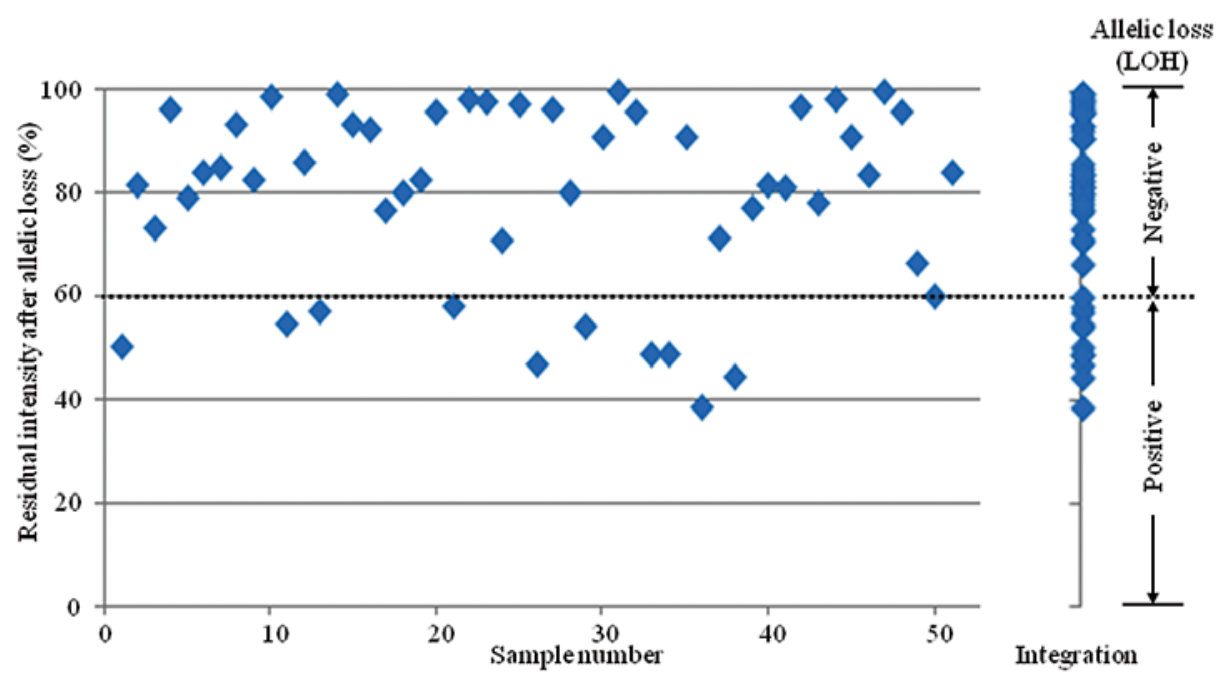

Figure 2. Distribution of LOH scores among 51 informative cases at rs3219489. The horizontal axis displays case number and the vertical axis LOH score. The LOH score of each case is plotted. When a score was $<0.6$ (40\% reduction in intensity of 1 allele from cancer), the case was judged as allelic loss (LOH positive). The right side shows an integration of all plots.

dominant condition (Table VI). On the other hand, those were detected in $8(36.4 \%)$ and $6(27.3 \%)$, respectively, of 22 cases with a $\mathrm{C}$-allele dominant condition. Thus, the ratio of cases with $\mathrm{G}: \mathrm{C} \rightarrow \mathrm{T}$ :A transversions among all cases with
KRAS mutations was calculated to be $36.4 \%$ in a $\mathrm{G}$-allele dominant condition and $75.0 \%$ in a $\mathrm{C}$-allele dominant condition. However, there was no significant difference between the allele dominant conditions. 


\section{Discussion}

To the best of our knowledge, this is the first extensive analysis of alterations of the $M U T Y H$ gene in sporadic CRC in an Asian population. Using DNA sequencing analyses of the MUTYH gene, 3 kinds of deleterious mutations, c. $35 \mathrm{C} \rightarrow \mathrm{T}$ p.Pro18Leu, c.55C $\rightarrow$ T p.Arg19X, and c.74G $\rightarrow$ A p.Gly25Asp, were detected in $3(3.0 \%)$ of $101 \mathrm{CRC}$ cases. All patients developed CRC at the age of 70 years or older, and had neither familial aggregation of CRC nor multiple colorectal polyps. However, these mutations detected in cancer DNA were also found in normal mucosal DNA of the same patient. Therefore, no somatic alteration in the MUTYH gene was observed.

Our results are the same as those of a previous study, which found no somatic MUTYH mutations in CRC cases (14). In this respect, somatic alterations of various repair genes, such as $M L H 1, M S H 2$ and $B R C A 2$, as well as others are considered to be rare events $(15,16)$. Sieber et al (8) found monoallelic germline mutations of the MUTYH gene in $6(3.1 \%)$ of 130 patients with multiple colorectal adenomas. Those 6 patients had characteristic features of developing only a few adenomas (mean of 4). Two missense mutations, c.35C $\rightarrow$ T p.Pro18Leu and c.74G $\rightarrow$ A p.Gly25Asp, were previously reported to be germline mutations based on an analysis of Japanese patients with adenomatous polyposis (17). In our study, 2 patients were confirmed to have the same compound heterozygous condition of MUTYH. However, they lacked characteristic features of MAP, such as multiple colorectal tumors, young onset of disease, and familial aggregation, and other distinctive clinical pathological features. These findings suggest that the phenotypes are rather diverse, even if the same mutated genotype of MUTYH exists. This notion is supported by Farrington's study, which showed that $36 \%$ of biallelic carriers of $M U T Y H$ mutations had no polyps (11). On the other hand, the present nonsense mutation, c.55C $\rightarrow \mathrm{T}$ p.Arg19X, has not been registered in HGMD or dbSNP of NCBI. Therefore, it is considered to be a novel mutation.

The MUTYH gene has been mapped to the short arm of chromosome 1, 1p34.3-p32.1 (5). Several cytogenetic and molecular genetic investigations of colorectal tumors including adenoma and cancer have shown that allelic loss of the short arm of chromosome 1 is genetically common, though its frequency varies from $12-84 \%(8,18-21)$. In the present study, allelic loss at the MUTYH locus was found in $10(19.6 \%)$ of the 51 informative cases, which is comparable to the previous data (Table IVA). A possible explanation is that we used few markers, which were strictly localized within 1 gene of interest, unlike others who studied a large number of genetic markers arranged widely in the $1 \mathrm{p}$ region. Therefore, with regard to the significance of allelic loss of the MUTYH locus, the present findings are considered to be more meaningful. Sieber et al (8) suggested that absence of the MUTYH gene may be not essential for colorectal tumorigenesis, because of the relatively low frequency of LOH of MUTYH in colorectal adenoma cases $(12.0 \%)$. However, allelic loss in the $1 p$ region including the MUTYH locus has been frequently observed in colorectal adenomas and cancer in a number of studies, suggesting its role in both, as well as late colorectal carcinogenesis (19-22).

There was also a significant association between state of allelic loss and the location of CRC occurrence (Table IVB).
Ten CRC cases with allelic loss showed occurrence in the left-sided colon and rectum. In contrast, a previous report indicated that MAP, which is caused by deleterious germline mutations of the MUTYH gene, has a characteristic of predominantly right-sided CRC with similarities to microsatellite unstable carcinomas, such as Lynch syndrome (23). An attractive hypothesis states that differences between germline mutations and somatic alterations in the same gene will lead to different phenotypes, though there is no direct supporting evidence at present. Our results are thought to be provoking and interesting in terms of a possible association between the genetic condition of MUTYH and carcinogenesis in the colon and rectum.

$\mathrm{G}: \mathrm{C} \rightarrow \mathrm{T}: \mathrm{A}$ transversion is considered to be a specific target for MUTYH. Therefore, our findings regarding the spectrum of $K R A S$ mutations in this series, especially in correlation with alterations of the MUTYH gene, are interesting. The overall detection rate of KRAS mutation was $32.7 \%$ (33/101) and the distribution of mutant types was similar to previous reports (Table V) $(24,25)$. Of 3 CRC cases among germline mutation carriers of $M U T Y H$, the presence of $\mathrm{G}: \mathrm{C} \rightarrow \mathrm{T}: \mathrm{A}$ somatic mutations (c.34G $\rightarrow \mathrm{T}$ p.Gly12Cys, c.35G $\rightarrow \mathrm{T}$ p.Gly12Val) in KRAS was confirmed in only 1 (33.3\%), which was not significantly different from the outcome of all target samples. $\mathrm{G}: \mathrm{C} \rightarrow \mathrm{T}: \mathrm{A}$ somatic transversion was detected in only $1(10.0 \%)$ of 10 CRC cases with allelic loss. In contrast, 9 (22.0\%) of 41 cases without allelic loss had $\mathrm{G}: \mathrm{C} \rightarrow \mathrm{T}$ :A somatic mutations. On the other hand, in the 51 samples subjected to $\mathrm{LOH}$ analysis, $\mathrm{G}: \mathrm{C} \rightarrow \mathrm{T}: \mathrm{A}$ somatic mutations were more frequently found as compared to all of the samples. However, there was no statistically significant difference between CRC cases with and without allelic loss.

Inevitably, the 51 cases in the $\mathrm{LOH}$ analysis were evenly heterozygous at rs 3219489 , thus they are considered to be a kind of monoallelic variant, which causes substitution of glutamine (Gln) for histidine (His) at the 324 residue of the MUTYH protein. As for $\mathrm{C}$-allele encoding histidine in this SNP site, some interesting reports have been presented. Ali et al indicated that the SNP (Gln324His) of MUTYH was partially impaired in regard to adenine removal (27). Furthermore, in an association study conducted in Japan, Tao et al showed that the T-G-A-C haplotype, which consists of IVS1+11C $\rightarrow \mathrm{T}(\mathrm{rs} 2275602)$, IVS6+35G $\rightarrow \mathrm{A}(\mathrm{rs} 112763487)$, IVS10 $-2 A \rightarrow G(r s 77542170)$, and $972 G \rightarrow C(r s 3219489)$ sequentially, was significantly associated with an increased risk of distal colon cancer (26). Therefore, cases with the heterozygous genotype including the C-allele at rs3219489 might be partially related to inactive base excision repair function and occur in a mutable predisposition. This speculation is strongly supported by a previous findings showing that the presence of $\mathrm{G}: \mathrm{C} \rightarrow \mathrm{T}: \mathrm{A}$ transversions in KRAS or APC was significantly more common in tumors with monoallelic MUTYH variants than in those with the wild-type genotype (28). Interestingly, we found that $\mathrm{G}: \mathrm{C} \rightarrow \mathrm{T}: \mathrm{A}$ transversions occurred more frequently in CRCs with a $\mathrm{C}$-allele dominant condition corresponding to His than in those with G-allele dominance corresponding to Gln (Table VI). Thus, these results indicate that quantitative allelic imbalance of the MUTYH gene may influence the function of adenine glycosylase. It will be necessary to clarify these interesting 
phenomena using functional analysis, such as the previous method advocated by Goto et al (29).

In the future, it is important to clarify the role of monoallelic variants in relation to mutable potential via either a complete or at least partial defect of base excision repair function using other genetic markers. Furthermore, it would be interesting to investigate possible germline mutations of regions not analyzed in this study as well as epigenetic changes of the promoter region of $M U T Y H$ in terms of possible contributions to carcinogenesis in sporadic CRC cases.

\section{Acknowledgements}

This study was supported by a Grant-in-Aid from the Japanese Ministry of Education, Science, Sports and Culture of Japan (22591497 and 23591974), and Grant-in-Aid from Hyogo College of Medicine (Nagahide Matsubara).

\section{References}

1. Tamura K, Utsunomiya J, Iwama T, et al: Mechanism of carcinogenesis in familial tumors. Int J Clin Oncol 9: 232-245, 2004.

2. Vogelstein B and Kinzler KW: Cancer genes and the pathways they control. Nat Med 10: 789-799, 2004.

3. Hung R, Hung RJ, Hall J, Brennan P and Boffetta P: Genetic polymorphisms in the base excision repair pathway and cancer risk: a HuGE review. Am J Epidemiol 162: 925-942, 2005.

4. Krokan HE, Nilsen H, Skorpen F, Otterlei M and Slupphaug G: Base excision repair of DNA in mammalian cells. FEBS Lett 476: 73-77, 2000.

5. Slupska MM, Baikalov C, Luther WM, Chiang JH, Wei YF and Miller JH: Cloning and sequencing a human homolog $(h M Y H)$ of the Escherichia coli mut $Y$ gene whose function is required for the repair of oxidative DNA damage. J Bacteriol 178: 3885-3892, 1996.

6. Shinmura K, Yamaguchi S, Saitoh T, et al: Adenine excisional repair function of MYH protein on the adenine:8-hydroxyguanine base pair in double-stranded DNA. Nucleic Acids Res 28: 4912-4918, 2000.

7. Al-Tassan N, Chmiel NH, Maynard J, et al: Inherited variants of $\mathrm{MYH}$ associated with somatic $\mathrm{G}: \mathrm{C} \rightarrow \mathrm{T}:$ A mutations, in colorectal tumors. Nat Genet 30: 227-232, 2002.

8. Sieber OM, Lipton L, Crabtree M, et al: Multiple colorectal adenomas, classic adenomatous polyposis, and germ-line mutations in $M Y H$. N Engl J Med 348: 791-799, 2003.

9. Miyaki M, Iijima T, Yamaguchi T, Hishima T, Tamura K, Utsunomiya $\mathrm{J}$ and Mori T: Germline mutations of the $M Y H$ gene in Japanese patients with multiple colorectal adenomas. Mutat Res 578: 430-433, 2005.

10. Jones S, Emmerson P, Maynard J, et al: Biallelic germline mutations in MYH predispose to multiple colorectal adenoma and somatic G:C $\rightarrow$ T:A mutations. Hum Mol Genet 11: 2964-2967, 2002.

11. Farrington SM, Tenesa A, Barnetson R, et al: Germline susceptibility to colorectal cancer due to base-excision repair gene defects. Am J Hum Genet 77: 112-119, 2005.

12. Saeki Y, Tamura K, Yamamoto Y, et al: Germline p53 mutation at codon 133 in a cancer-prone family. $\mathrm{J}$ Mol Med 75: 50-56, 1997.
13. Jernvall P, Mäkinen MJ, Karttunen TJ, Mäkelä J and Vihko P: Loss of heterozygosity at 18q21 is indicative of recurrence and therefore poor prognosis in a subset of colorectal cancers. Br J Cancer 79: 903-908, 1999.

14. Halford SER, Rowan AJ, Lipton L, et al: Germline mutations but not somatic changes at the $M Y H$ locus contribute to the pathogenesis of unselected colorectal cancers. Am J Pathol 162: 1545-1548, 2003.

15. Kowalski LD, Mutch DG, Herzog TJ, Rader JS and Goodfellow PJ: Mutational analysis of MLH1 and MSH2 in 25 prospectivelyacquired RER ${ }^{+}$endometrial cancers. Genes Chromosomes Cancer 18: 219-227, 1997.

16. Miki Y, Katagiri T, Kasumi F, Yoshimoto T and Nakamura Y: Mutation analysis in the BRCA2 gene in primary breast cancers. Nat Genet 13: 245-247, 1996.

17. Yanaru-Fujisawa R, Matsumoto T, Ushijima Y, et al: Genomic and functional analyses of MUTYH in Japanese patients with adenomatous polyposis. Clin Genet 73: 545-553, 2008

18. Vogelstein B, Fearon ER, Kern SE, et al: Allelotype of colorectal carcinomas. Science 244: 207-211, 1989.

19. Couturier-Turpin MH, Esnous C, Louvel A, Poirier Y and Couturier D: Chromosome 1 in human colorectal tumors. Cytogenetic research on structural changes and their significance. Hum Genet 88: 431-438, 1992.

20. Bardi G, Sukhikh T, Pandis N, Fenger C, Kronborg O and Heim S: Karyotypic characterization of colorectal adenocarcinomas. Genes Chromosomes Cancer 12: 97-109, 1995.

21. Praml C, Finke LH, Herfarth C, Schlag P, Schwab M and Amler L: Deletion mapping defines different regions in 1p34.2pter that may harbor genetic information related to human colorectal cancer. Oncogene 11: 1357-1362, 1995.

22. Thorstensen L, Qvist H, Heim S, et al: Evaluation of 1p losses in primary carcinomas, local recurrence and peripheral metastases from colorectal cancer patients. Neoplasia 2: 514-522, 2000.

23. Nielsen M, de Miranda NF, van Puijenbroek M, et al: Colorectal carcinomas in MUTYH-associated polyposis display histopathological similarities to microsatellite unstable carcinomas. BMC Cancer 9: 184, 2009.

24. Neumann J, Zeidl-Eberhart E, Kirchnet T and Jung A: Frequency and type of KRAS mutations in routine diagnostic analysis of metastatic colorectal cancer. Pathol Res Pract 205: 858-862, 2009.

25. Deschoolmeester V, Boeckx C, Baay M, et al: KRAS mutation detection and prognostic potential in sporadic colorectal cancer using high-resolution melting analysis. Br J Cancer 103: 1627-1636, 2010.

26. Tao H, Shinmura K, Suzuki M, et al: Association between genetic polymorphisms of the base excision repair gene $M U T Y H$ and increased colorectal cancer risk in a Japanese population. Cancer Sci 99: 355-360, 2008.

27. Ali M, Kim H, Cleary S, Cupples C, Gallinger S and Bristow R: Characterization of mutant MUTYH proteins associated with familial colorectal cancer. Gastroenterology 135: 499-507, 2008.

28. Kambara T, Whitehall VL, Spring KJ, et al: Role of inherited defects of $M Y H$ in the development of sporadic colorectal cancer. Genes Chromosomes Cancer 40: 1-9, 2004.

29. Goto M, Shinmura K, Nakabeppu Y, Tao H, Yamada H, Tsuneyoshi T and Sugimura H: Adenine DNA glycosylase activity of 14 human MutY homolog (MUTYH) variant proteins found in patients with colorectal polyposis and cancer. Hum Mutat 31: E1861-E1874, 2010. 